\title{
Expression of pituitary tumor-transforming 2 in human glioblastoma cell lines and its role in glioblastoma tumorigenesis
}

\author{
YUNBAO GUO $^{1}$, YIMENG SHAO ${ }^{2}$, JING CHEN $^{3}$, SONGBAI XU $^{1}$, XINGDONG ZHANG $^{4}$ and HAIYAN LIU ${ }^{4}$ \\ ${ }^{1}$ Department of Neurosurgery, The First Hospital of Jilin University, Changchun, Jilin 130021; \\ ${ }^{2}$ Operating Room, Central Hospital of Changchun, Changchun, Jilin 130011; \\ ${ }^{3}$ Department of Orthopedics, China-Japan Union Hospital of Jilin University, Changchun, Jilin 130033; \\ ${ }^{4}$ Department of Anatomy, College of Basic Medical Sciences, Jilin University, Changchun, Jilin 130021, P.R. China
}

Received May 19, 2015; Accepted January 11, 2016

DOI: $10.3892 / e t m .2016 .3159$

\begin{abstract}
The present study aimed to investigate the association between the expression of pituitary tumor-transforming 2 (PTTG2), and cell proliferation, invasion and apoptosis in glioblastoma. The U251 human glioblastoma cell line was transfected with the pcDNA-PTTG2 and small interfering (si)RNA-PTTG2 plasmids using Lipofectamine 2000. The expression of PTTG2 in U251 glioblastoma cells was determined using reverse transcription-quantitative polymerase chain reaction (RT-qPCR) and western blot analysis. The association between PTTG2 expression, and cell proliferation, invasion and apoptosis in vitro were investigated using an MTT assay, Matrigel Transwell assay and flow cytometry combined with Annexin V/propidium iodide staining, respectively. RT-qPCR and western blot analysis demonstrated that PTTG2 mRNA and protein expression were significantly overexpressed and significantly suppressed following transfection with pcDNA-PTTG2 and short interfering RNA (siRNA)-PTTG2 plasmids, respectively $(\mathrm{P}<0.05)$. In addition, the cell proliferation rate and invasive cell number in cells with overexpressed PTTG2 were significantly higher compared with cells in the untreated group, and the invasive cell number in the siRNA-PTTG2 group was significantly lower than the untreated group $(\mathrm{P}<0.05)$. Flow cytometry analysis demonstrated that, compared with the untreated group, the quantity of apoptotic cells in PTTG2 overexpression group was significantly reduced, and the quantity of apoptotic cells in the siRNA-PTTG2 group was increased. Similar results were obtained with regards to the expression level of caspase- 3 . The results of the present study indicate that PTTG2 overexpression promotes cell proliferation and invasion during glioblastoma
\end{abstract}

Correspondence to: Dr Haiyan Liu, Department of Anatomy, College of Basic Medical Sciences, Jilin University, 126 Xinmin Street, Changchun, Jilin 130021, P.R. China

E-mail: haiyan@jlu.edu.cn

Key words: glioblastoma, pituitary tumor-transforming 2, MTT assay, Matrigel transwell assay, flow cytometry progression. In addition, the results suggest that PTTG2 overexpression inhibits cell apoptosis in glioblastoma by affecting caspase-3-dependent signaling pathways. It can therefore be suggested that PTTG2 may serve as a novel therapeutic target for treating glioblastoma.

\section{Introduction}

Gliomas are a subtype of primary brain tumor that are known to be aggressive and highly invasive (1). The most aggressive glioma manifestation is glioblastoma; its invasive nature contributes towards the ineffectiveness of current therapeutic strategies, such as neurosurgery, radiation therapy and chemotherapy (2). Although efforts have been made to advance cancer diagnosis and treatment, the impact of these advances on the clinical outcome and prognosis for patients with glioblastoma has not improved $(3,4)$. Therefore, it is of great importance to elucidate the molecular mechanisms underlying glioblastoma in order to further explore effective treatments for the disease.

The pituitary tumor transforming gene (PTTG) has been demonstrated to serve a role in tumor initiation and progression, including control of mitosis, cell transformation and DNA repair (5). A whole-genome expression profiling has revealed that PTTG1 and PTTG2 are regulated in high-grade glioma, and may be involved in its malignant progression (6). Moreover, PTTG1 and PTTG2 have been identified in two glioblastoma-associated stromal cell subtypes with different carcinogenic properties present in the stroma of carcinomas (7). In addition, induced PTTG1 expression is correlated with invasiveness and poor prognosis in glioma patients (8), and increasing evidence confirms a crucial role of PTTG1 in cell physiology and tumorigenesis (9). However, the role of PTTG2 expression in glioblastoma tumorigenesis remains uncertain. Thus, there is a requirement for increased understanding of the role of PTTG2 in glioblastoma progression in order to determine novel target molecules.

In the present study, PTTG2 was overexpressed and suppressed in the U251 human glioblastoma cell line following transfection with pcDNA-PTTG2 and short interfering RNA (siRNA)-PTTG2 plasmids, respectively. Following this, cell proliferation, invasion and the apoptotic capacity were analyzed in vitro. The current study aimed to investigate the 
association between PTTG2 expression and glioblastoma tumorigenesis, in order to provide further insight into the mechanisms underlying glioblastoma disease and to determine a theoretical foundation for therapeutically targeting PTTG2 in the treatment of glioblastoma.

\section{Materials and methods}

Cell culture and plasmid transfection. U251 human glioblastoma cell lines were obtained from the Institute of Biochemistry and Cell Biology, Chinese Academy of Sciences (Shanghai, China) and cultured in Dulbecco's modified Eagle's medium (DMEM) containing 10\% heat-inactivated fetal bovine serum (Welgene Biotech, Co., Ltd., Taiwan). At $24 \mathrm{~h}$ prior to transfection, $2 \times 10^{5} \mathrm{U} 251$ cells per well were plated into 6-well plates and grown until they were $50-80 \%$ confluent. Plasmids pcDNA-PTTG2 and siRNA-PTTG2 were purchased from the American Type Culture Collection (Manassas, VA, USA) and were transfected into U251 cells with Lipofectamine 2000 reagent (Invitrogen; Thermo Fisher Scientific, Inc., Waltham, MA, USA) for $24 \mathrm{~h}$ at room temperature, according to the manufacturer's instructions. U251 cells transfected with a pcDNA vector were used as the control (untreated) group. Cells from each group were incubated at room temperature using normal DMEM media for $4 \mathrm{~h}$ and were harvested after $48 \mathrm{~h}$ for further detection.

Reverse transcription-quantitative polymerase chain reaction (RT-qPCR) analysis. Total RNA was extracted from U251 cells from each group using TRIzol reagent (Invitrogen; Thermo Fisher Scientific, Inc.) following treatment with TURBO DNase (Ambion; Thermo Fisher Scientific, Inc.), and a spectrophotometer (NanoDrop 2000; Thermo Fisher Scientific, Inc.) was used to measure the quality of RNA. Next, $1 \mu 1 \mathrm{cDNA}$ generated from $1 \mu \mathrm{g}$ RNA was obtained by RT using the PrimeScript RT Master Mix kit (RR036A; Takara Biotechnology, Co., Ltd., Dalian, China). RT-qPCR was then performed using Superscript III (Invitrogen; Thermo Fisher Scientific, Inc.) and the expression level of PTTG2 was determined using the TaqMan Gene Expression Assay (Hs00747713_sH; Applied Biosystems; Thermo Fisher Scientific, Inc.). Reactions were conducted at $95^{\circ} \mathrm{C}$ for $10 \mathrm{~min}$ followed by 40 cycles of $95^{\circ} \mathrm{C}$ for $15 \mathrm{sec}, 60^{\circ} \mathrm{C}$ for $1 \mathrm{~min}$ and a final dissociation stage of a gradient of $30-99^{\circ} \mathrm{C}$ using an ABI 7500 RT-PCR system (Applied Biosystems; Thermo Fisher Scientific, Inc.). Each sample was analyzed in triplicate. Finally, the $2^{-\Delta \Delta C q}$ method (10) was used to calculate the expression level of PTTG2 according to the expression of glyceraldehyde 3-phosphate dehydrogenase.

Western blot analysis. The expression of PTTG2 and caspase- 3 at the protein level were measured using western blot analysis. Cell lysates were prepared using a radioimmunoprecipitation assay buffer (Roche Diagnostics, Basel, Switzerland). Protein concentrations were examined by a Bradford assay (Bio-Rad Laboratories, Inc., Madrid, Spain). Protein bands were distinguished by sodium dodecyl sulfate-polyacrylimide gel electrophoresis (Sigma-Aldrich, St. Louis, MO, USA and Bio-Rad Laboratories, respectively) at $100 \mathrm{~V}$ for $2 \mathrm{~h}$ and subsequently transferred to nitrocellulose membranes (GE
Healthcare Life Sciences, Chalfont, UK). The membranes were blocked with 5\% nonfat dry milk for $1 \mathrm{~h}$, then the membrane was probed using specific rabbit primary antibodies against human caspase-3, PTTG2 and actin (all 1:1,000; Abgent, Inc., San Diego, CA, USA) overnight at $4^{\circ} \mathrm{C}$ and incubated with horseradish peroxidase-conjugated secondary antibody (1,5,000; all Santa Cruz Biotechnology, Inc., Dallas, TX, USA). The fold change in specific protein expression was determined using $\beta$-actin expression as a loading control. Densitometric measurements of the bands were analyzed using the Kodak 2000R imaging system (Kodak, Rochester, NY, USA).

3-(4,5-dimethylthiazol-2-yl)-2,5-diphenyltetrazolium bromide (MTT) assay. U251 cells ( $2 \times 10^{4}$ cells/well) from each group were seeded on 96-well plates. Next, $20 \mu 10.5 \mathrm{mg} / \mathrm{ml}$ MTT (Sigma-Aldrich) was added to each well after 0, 24, 48, 72 and $96 \mathrm{~h}$ of transfection. Next, the plates were incubated for $4 \mathrm{~h}$ at $37^{\circ} \mathrm{C}$, followed by the addition of $200 \mu 1$ dimethyl sulfoxide into each well and further incubation for $30 \mathrm{~min}$. Finally, the optical density was read at a wavelength of $570 \mathrm{~nm}$ with a VERSAmax microplate reader (Molecular Devices LLC, Sunnyvale, CA, USA). The experiment was performed in triplicate. The cell proliferation/viability was calculated using the obtained numerical values using the following equation: Cell viability $=[$ optical density (OD) value of test group - OD value of blank group] / (OD value of control group - OD value of blank group).

Matrigel Transwell assay. The invasion of glioblastoma cells in vitro was measured using a Transwell chamber with a Matrigel-coated $(0.78 \mathrm{mg} / \mathrm{ml})$ upper membrane, which contained a 24-well insert with $8 \mathrm{~mm}$ pore size (all BD Biosciences, Franklin Lanes, NJ, USA). The transfected U251 cells were starved for $24 \mathrm{~h}$ and harvested. Next, $500 \mu \mathrm{l}$ cells $\left(1 \times 10^{5}\right.$ cells $\left./ \mathrm{ml}\right)$ from each group were added to the upper insert of the chamber containing serum-free media (Sigma-Aldrich). Meanwhile, the lower well of the chamber was filled with DMEM with $10 \%$ bovine serum albumin (Sigma-Aldrich). Following $12 \mathrm{~h}$ of incubation, $70 \%$ ice-cold ethanol was used to fix the cells that had invaded the membrane and $0.1 \%$ crystal violet (Sigma-Aldrich) was used to stain the cells for $15 \mathrm{~min}$ at room temperature. Each determination was assayed in triplicate. Finally, 8 random high-power fields per chamber were counted under a Leica DM2500 light microscope (magnification, x100; Leica Microsystems GmbH, Wetzlar, Germany) and the sum of the invasive cell number was calculated to analyze the invasive ability.

Apoptotic analysis using Annexin V/propidium iodide staining and flow cytometry. The apoptotic rate of U251 cells was detected using flow cytometry. Analysis of Annexin V was examined using the Annexin V-fluorescein isothiocyanate (FITC) apoptosis detection kit (Beijing Biosea Biotechnology, Co., Ltd., Beijing, China), in accordance with the manufacturer's instructions. U251 cells $\left(5 \times 10^{6}\right.$ cells/well $)$ were resuspended and allowed to attach overnight. Next, cells were isolated with trypsin (Sigma-Aldrich), washed with ice-cold phosphate-buffered saline and resuspended in $96 \mu \mathrm{l}$ Annexin V binding buffer (BD Biosciences). A total of $1 \mu \mathrm{l}$ Annexin V-FITC and $5 \mu \mathrm{l}$ propidium iodide solution (both 
A

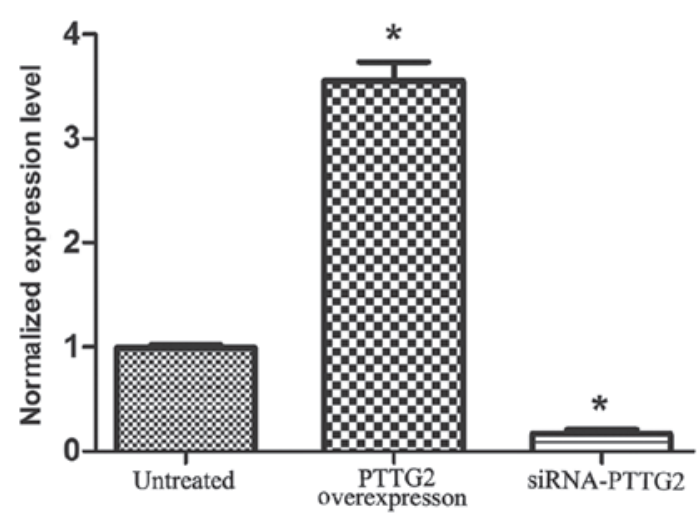

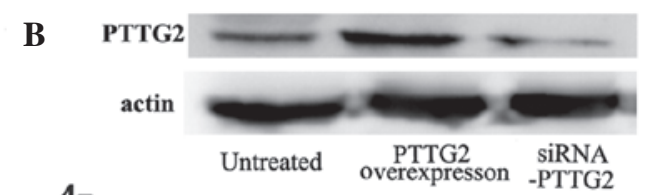

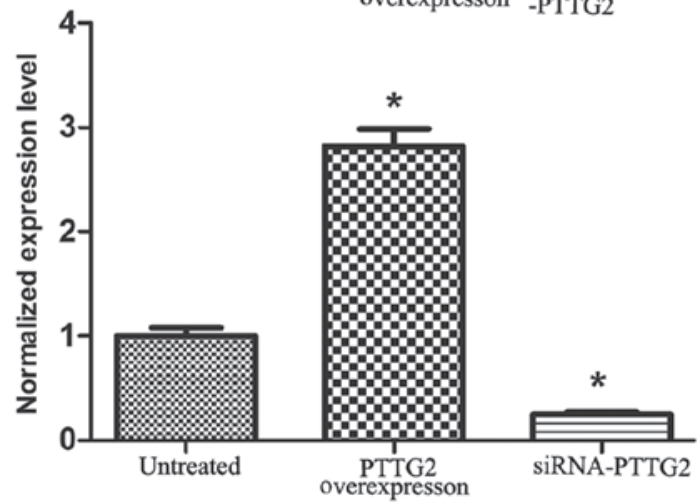

Figure 1. Expression levels of PTTG2. (A) The expression level of PTTG2 analyzed by reverse transcription-polymerase chain reaction. (B) The expression level of PTTG2 analyzed by western blotting. Error bars indicate the mean \pm standard deviation. "P $<0.05$ vs. untreated group. PTTG2, pituitary tumor-transforming 2; siRNA, small interfering RNA.

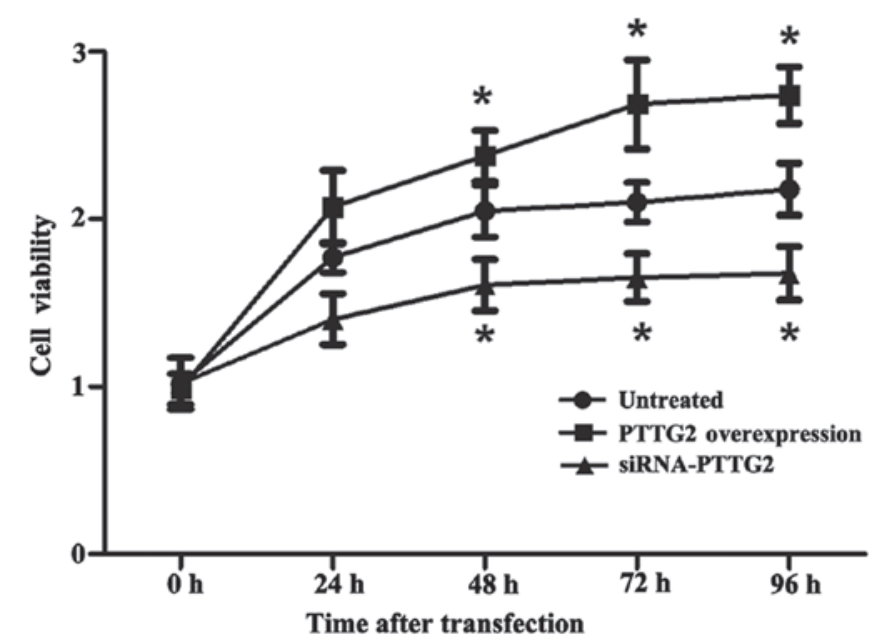

Figure 2. Cell proliferation/viability over time. Error bars indicate the mean \pm standard deviation. ${ }^{*} \mathrm{P}<0.05$ vs. untreated group. PTTG2, pituitary tumor-transforming 2; siRNA, small interfering RNA.

Sigma-Aldrich) was then added to cells, followed by incubation away from light and on ice for $15 \mathrm{~min}$. The cells were analyzed at $488 \mathrm{~nm}$ by flow cytometry (BD FACSAria; BD Biosciences) and the obtained numerical values were analyzed using CellQuest version 3.0 software (BD Biosciences). A dual-color flow cytometric method (11) was used to count the Annexin V-positive cells as apoptotic cells.

Statistical analysis. The normal distribution of all collected data was determined using the one-sample Kolmogorov-Smirnov test. Enumeration data were analyzed using the $\chi^{2}$ or rank-sum test. Measurement data was analyzed by Student's t-test (for two groups) or one-way analysis of variance (for more than three groups). A post-hoc Tukey's test was used to analyze comparisons between groups. All variable data were analyzed using SPSS 13.0 software (SPSS, Inc., Chicago, IL, USA). P $<0.05$ was considered to indicate a statistically significantly difference.

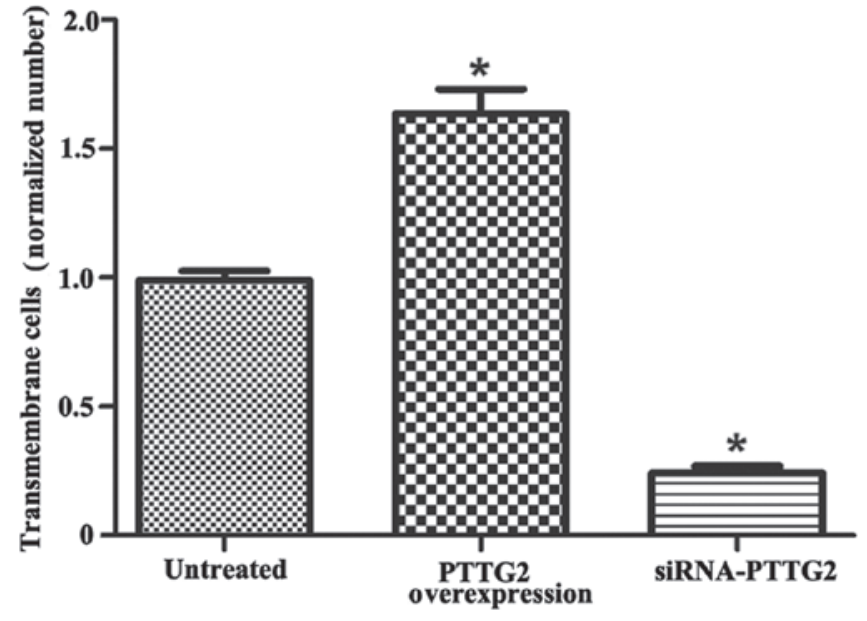

Figure 3. Role of PTTG2 expression in cell invasion. Error bars indicate the mean \pm standard deviation. "P $<0.05$ vs. untreated group. PTTG2, pituitary tumor-transforming 2; siRNA, small interfering RNA.

\section{Results}

PTTG2 was successfully overexpressed and knocked down in U251 cells. As presented in Fig. 1, the expression of PTTG2 mRNA and protein were determined. The results demonstrated that the expression of PTTG2 mRNA and protein (Fig. 1B) in U251 cells in the PTTG2 overexpression group significantly increased in comparison with the untreated group $(\mathrm{P}<0.05)$, whereas the expression was significantly reduced in the siRNA-PTTG2 interference group in comparison with the untreated group $(\mathrm{P}<0.05)$. This indicates that PTTG2 was successfully overexpressed and suppressed in the study.

PTTG2 overexpression increases cell viability. An MTT assay was performed to analyze the proliferation of U251 cells in each group in an experimental period of $96 \mathrm{~h}$ subsequent to transfection. As presented in Fig. 2, the cell proliferation/viability of the PTTG2 overexpression group significantly 


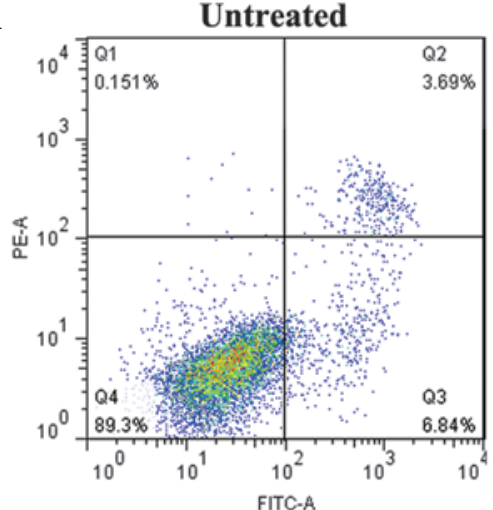

B

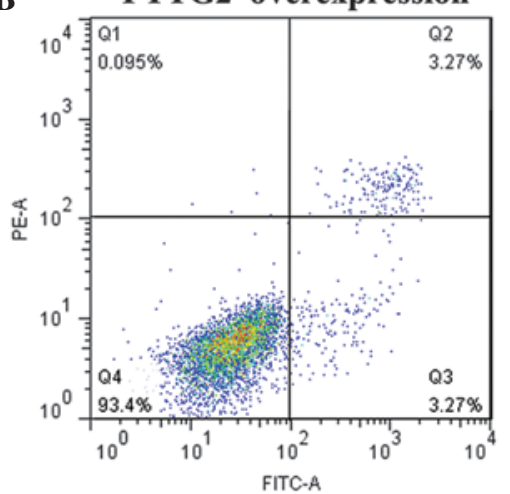

C

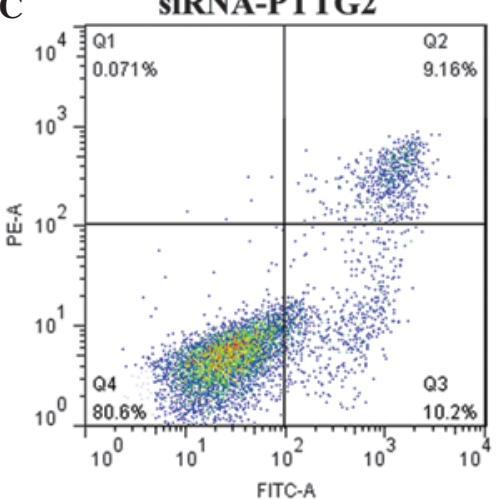

D

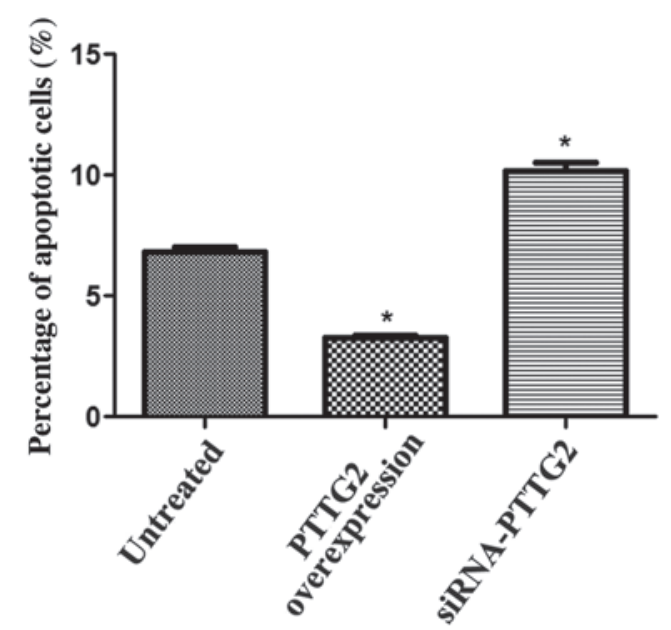

$\mathbf{E}$

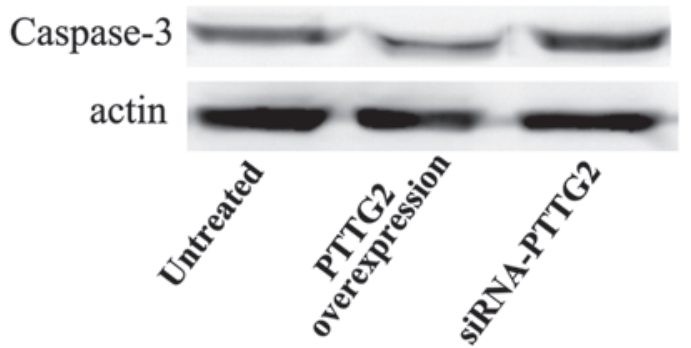

Figure 4. Association between PTTG2 expression and cell apoptosis. Apoptosis cells in the (A) untreated, (B) PGGT2 overexpression and (C) siRNA-PTTG2 groups were analyzed by flow cytometry. (D) Quantitative analysis demonstrated that PTTG2 overexpression significantly decreased the percentage of apoptotic cells, whereas the PTTG2 knockdown significantly increased the percentage of apoptotic cells. (E) The expression level of caspase-3 was analyzed by western blotting. Error bars indicate the mean \pm standard deviation. ${ }^{*} \mathrm{P}<0.05$ vs. untreated group. PR, phycoerythrin; FITC, fluorescein isothiocyanate; PTTG2, pituitary tumor-transforming 2; siRNA, small interfering RNA.

increased over time in comparison with the untreated group $(\mathrm{P}<0.05)$, whilst cell proliferation/viability was reduced in the siRNA-PTTG2 interference group in comparison with the untreated group $(\mathrm{P}<0.05)$.

PTTG2 overexpression promotes cell invasion. Fig. 3 displays the cell invasion ability of each group. According to the sum of 8 random high-power fields from each group, the invasive cell number in the PTTG2 overexpression group was significantly higher than that of untreated group $(1.63$ times higher; $\mathrm{P}<0.05)$. Meanwhile, the invasive cell number in the siRNA-PTTG2 interference group was significantly reduced in comparison to the untreated group ( $28 \%$ of untreated group; $\mathrm{P}<0.05)$.

PTTG2 overexpression reduces cell apoptosis. Flow cytometry was used to analyze the level of apoptotic cells in each group. The results demonstrated that, compared with the untreated group, the number of apoptotic cells in the PTTG2 overexpression group was significantly reduced $(\mathrm{P}<0.05$; Fig. $4 \mathrm{~A}$ and $\mathrm{B})$ while the number of apoptotic cells in the siRNA-PTTG2 interference group was markedly increased (Fig. 4A and C). In addition, the expression level of caspase- 3 was determined to verify the association between PTTG2 expression and cell apoptosis. The expression level of caspase-3 was significantly reduced $(\mathrm{P}<0.05)$ in the $\mathrm{PTTG} 2$ overexpression group and markedly reduced in the siRNA-PTTG2 interference group, when compared with the untreated group (Fig. 4D).

\section{Discussion}

Glioblastoma multiforme is an aggressive cancer that has the highest rate of mortality among all malignant brain tumors (12). Therefore, there is an urgent requirement to identify novel target molecules to which more effective therapeutic approaches can be developed. In the current study, the results demonstrated that PTTG2 expression was strongly associated with cell proliferation and invasion in the U251 human glioblastoma cell line. Furthermore, PTTG2 was observed to induce cell apoptosis in U251 cells. Together, these results indicate that PTTG2 is likely to be associated with the progression of glioblastoma.

PTTG2 belongs to PTTG family, which is highly expressed in proliferating cells and serves an important role in mediating tumorigenic functions in a number of tumors (13). A previous study used siRNA interference to reveal that PTTG has key effects on cell proliferation, cycle and apoptosis in U251 glioma cells (14). In addition, Ishikawa et al (15) reported that PTTG could induce angiogenesis by targeting basic fibroblast 
growth factor to promote tumor progression. Angiogenesis is, therefore, considered to be a key determinant and rate-limiting step in tumor growth and metastatic spread (15).

PTTG2 is understood to share a high sequence homology with PTTG1 (16). Furthermore, overexpression of PTTG1 has been reported to induce c-Myc expression and consequently result in increased levels of cell proliferation (17). In the present study, the cell proliferation/viability in the PTTG2 overexpression group significantly increased over time. As a result, it is proposed that PTTG2 may serve a role in the regulation of cell growth in glioblastoma.

Furthermore, invasiveness is a key molecular mechanism that influences the malignant characteristics of gliomas (2). Méndez-Vidal et al (16) verified that PTTG2 serves an important role in cell adhesion and may serve a potential role in cell invasion. The study confirmed that knockdown of PTTG2 not only results in concomitant downregulation of E-cadherin, but also leads to the induction of epithelial-to-mesenchymal transition (EMT) and elevates vimentin expression levels. In addition, Canel et al (18) demonstrated that E-cadherin mediates homophilic cell adhesion and that a loss of its function may promote tumour invasion. Misregulated E-cadherin expression is also observed as an aggressive brain tumor phenotype in brain cancer, including glioblastoma (19). In addition, EMT has been demonstrated to provide motility and invasive ability to cancer cells during the progression of a number of types of human cancer (20), and vimentin intermediate filaments have been recognized as an essential component involved in cell invasion in lung cancer (21).

Zhou et al (22) suggested that vimentin expression correlates with cell shape and motility in U-373 MG glioblastoma cells. In the present study, the number of invasive cells in the PTTG2 overexpression group was significantly increased compared with that in the untreated group, and the number of invasive cells in the siRNA-PTTG2 interference group was significantly reduced in comparison with the untreated group. Therefore, the results suggest that PTTG2 may promote cell invasion in U251 glioblastoma cells by causing deregulation of E-cadherin or inhibiting EMT and reducing vimentin expression levels.

In order to study the effect of PTTG2 on apoptosis, the association between PTTG2 and caspase-3 was investigated. The results demonstrated that the expression level of caspase- 3 was reduced as PTTG2 expression increased, which was also observed in a study by Méndez-Vidal et al (16), indicating that PTTG2 may promote cell apoptosis via the activation of caspase-3. In addition, caspase-3, a widely accepted hallmark of programmed cell death, is able to cleave a broad range of cellular substrates and promote activation of DNA endonuclease (23); thus the activation of caspases-3 is a key molecular stage in apoptotic cell death in glioblastoma multiforme. Huang et al (24) demonstrated that curcuminoids can also suppress cell growth and induce apoptosis in GBM 8401 cells through affecting caspase-3-dependent signaling pathways. In the present study, flow cytometry results demonstrated that the number apoptotic cells in the PTTG2 overexpression group were significantly reduced in comparison with the untreated group, while the number of apoptotic cells in the siRNA-PTTG2 interference group were reduced in comparison with the untreated group. Thus, it can be hypothesized that PTTG2 induces cell apoptosis in U251 glioblastoma cells via caspase-3-dependent signaling pathways.

In conclusion, the current study indicates that PTTG2 overexpression can promote cell proliferation and invasion during the development of glioblastoma. Furthermore, PTTG2 overexpression may inhibit cell apoptosis in glioblastoma via caspase-3-dependent signaling pathways. Together, these observations provide an insight into the role of PTTG2 in glioblastoma tumorigenesis and suggest that PTTG2 may serve as a novel therapeutic target for the disease.

\section{References}

1. Maher EA, Furnari FB, Bachoo RM, Rowitch DH, Louis DN, Cavenee WK and DePinho RA: Malignant glioma: Genetics and biology of a grave matter. Genes Dev 15: 1311-1333, 2001.

2. Rao JS: Molecular mechanisms of glioma invasiveness: The role of proteases. Nat Rev Cancer 3: 489-501, 2003

3. Stewart LA: Chemotherapy in adult high-grade glioma: A systematic review and meta-analysis of individual patient data from 12 randomised trials. Lancet 359: 1011-1018, 2002

4. Stupp R, Mason WP, van den Bent MJ, Weller M, Fisher B, Taphoorn MJ, Belanger K, Brandes AA, Marosi C, Bogdahn U, et al; European Organisation for Research and Treatment of Cancer Brain Tumor and Radiotherapy Groups; National Cancer Institute of Canada Clinical Trials Group: Radiotherapy plus concomitant and adjuvant temozolomide for glioblastoma. N Engl J Med 352: 987-996, 2005.

5. Boelaert K, McCabe CJ, Tannahill LA, Gittoes NJ, Holder RL, Watkinson JC, Bradwell AR, Sheppard MC and Franklyn JA: Pituitary tumor transforming gene and fibroblast growth factor-2 expression: Potential prognostic indicators in differentiated thyroid cancer. J Clin Endocrinol Metab 88: 2341-2347, 2003.

6. Yang P, Yan W, Zhang W, You G, Bao ZS and Jiang T: Whole-genome messenger RNA profiling reveals genes involved in malignant progression of glioma. Zhonghua Yi Xue Za Zhi 93: 5-7, 2013 (In Chinese)

7. Clavreul A, Etcheverry A, Tétaud C, Rousseau A, Avril T, Henry $\mathrm{C}$, Mosser $\mathrm{J}$ and Menei P: Identification of two glioblastoma-associated stromal cell subtypes with different carcinogenic properties in histologically normal surgical margins. J Neurooncol 122: 1-10, 2015.

8. Genkai N, Homma J, Sano M, Tanaka R and Yamanaka R: Increased expression of pituitary tumor-transforming gene (PTTG)-1 is correlated with poor prognosis in glioma patients. Oncol Rep 15: 1569-1574, 2006.

9. Vlotides G, Eigler T and Melmed S: Pituitary tumor-transforming gene: Physiology and implications for tumorigenesis. Endocr Rev 28: 165-186, 2007.

10. Livak KJ and Schmittgen TD: Analysis of relative gene expression data using real-time quantitative PCR and the $2-\Delta \Delta \mathrm{Ct}$ method. Methods 25: 402-408, 2001.

11. Porra V, Bernaud J, Gueret P, Bricca P, Rigal D, Follea G and Blanchard D: Identification and quantification of fetal red blood cells in maternal blood by a dual-color flow cytometric method: evaluation of the Fetal Cell Count kit. Transfusion 47: 1281-1289, 2007.

12. Krex D, Klink B, Hartmann C, von Deimling A, Pietsch T, Simon M, Sabel M, Steinbach JP, Heese O and Reifenberger G, et al: Long-term survival with glioblastoma multiforme. Brain 130: 2596-2606, 2007.

13. Bradshaw C and Kakar SS: Pituitary tumor transforming gene: An important gene in normal cellular functions and tumorigenesis. Histol Histopathol 22: 219-226, 2007.

14. Song-Bai X, Hong-Guang Z, Gang Z, Zuo X, Hong-Quan Y and Shu-Hong K: The effect of PTTG gene silenced by RNAi on inhibition of human glioblastoma U251 cells. Chin J Gerontol 7: 645-647, 2008

15. Ishikawa H, Heaney AP, Yu R, Horwitz GA and Melmed S: Human pituitary tumor-transforming gene induces angiogenesis. J Clin Endocrinol Metab 86: 867-874, 2001.

16. Méndez-Vidal C, Gámez-Del Estal MM, Moreno-Mateos MA, Espina-Zambrano ÁG, Torres B and Pintor-Toro JA: PTTG2 silencing results in induction of epithelial-to-mesenchymal transition and apoptosis. Cell Death Dis 4: e530, 2013. 
17. Pei L: Identification of c-myc as a down-stream target for pituitary tumor-transforming gene. J Biol Chem 276: 8484-8491, 2001.

18. Canel M, Serrels A, Frame MC and Brunton VG: E-cadherin-integrin crosstalk in cancer invasion and metastasis. J Cell Sci 126: 393-401, 2013.

19. Lewis-Tuffin LJ, Rodriguez F, Giannini C, Scheithauer B, Necela BM, Sarkaria JN and Anastasiadis PZ: Misregulated E-cadherin expression associated with an aggressive brain tumor phenotype. PLoS One 5: e13665, 2010.

20. Nieto MA: The ins and outs of the epithelial to mesenchymal transition in health and disease. Annu Rev Cell Dev Biol 27: 347-376, 2011.

21. Kidd ME, Shumaker DK and Ridge KM: The role of vimentin intermediate filaments in the progression of lung cancer. Am J Respir Cell Mol Biol 50: 1-6, 2014.
22. Zhou R and Skalli O: TGF- $\alpha$ differentially regulates GFAP, vimentin, and nestin gene expression in U-373 MG glioblastoma cells: Correlation with cell shape and motility. Exp Cell Res 254: 269-278, 2000.

23. Ray SK, Patel SJ, Welsh CT, Wilford GG, Hogan EL and Banik NL: Molecular evidence of apoptotic death in malignant brain tumors including glioblastoma multiforme: Upregulation of calpain and caspase-3. J Neurosci Res 69: 197-206, 2002.

24. Huang TY, Tsai TH, Hsu CW and Hsu YC: Curcuminoids suppress the growth and induce apoptosis through caspase-3-dependent pathways in glioblastoma multiforme (GBM) 8401 cells. J Agric Food Chem 58: 10639-10645, 2010. 